\title{
Invasive Pulmonary Aspergillosis (IPA) Post Hematopoietic Stem Cell Transplant (HSCT): Is Immunological Dysfunction Play a Role?
}

\author{
Ashok Kuwal ${ }^{*}$, Naveen Dutt ${ }^{2}$, Nishant Chauhan ${ }^{2}$ \\ 'Department of Respiratory Medicine, Pacific Institute of Medical Sciences, India
}

${ }^{2}$ Department of Pulmonary Medicine, AlIMS Jodhpur, India

Article Info

\section{Article Notes}

Received: July 06, 2018

Accepted: August 01, 2018

\section{${ }^{*}$ Correspondence:}

Dr. Ashok Kuwal, 1007, gyan nagar, sector-4, Hiran magri, Udaipur, Rajasthan, 313002, India; Contact No: 91+9529554215, Email: kuwal.dr@gmail.com

(c) 2018 Kuwal A. This article is distributed under the terms of the Creative Commons Attribution 4.0 International License.

\section{Abstract}

Invasive pulmonary aspergillosis (IPA) is a major cause of morbidity and mortality among patients undergoing hematopoietic stem cell transplant (HSCT). The majority of cases are detected during the period of neutropenia (following conditioning regimen) or immunosuppression (treatment of graft versus host disease). Development of IPA after one-year post- HSCT is extremely rare. Here, we report a case of a 43-year-old male who developed IPA two years after an allogenic stem cell transplant and 406 days after stopping the immunosuppressive medication.

\section{Introduction}

Invasive pulmonary aspergillosis (IPA) is potentially a life threatening invasive fungal disease caused by Aspergillus spp. Despite advances in therapy, it is associated with significant morbidity and mortality. Risk factors for IPA are prolonged neutropenia, immunosuppressive therapy, solid organ transplant, hematopoietic stem cell transplant (HSCT), haematological malignancies, critical illness in intensive care units and chronic lung diseases ${ }^{1}$.

Most of the cases of IPA in patients of post-HSCT are seen either following HSCT or during the treatment of graft versus host disease (GVHD). Diagnosis of IPA beyond two years post HSCT is exceptionally rare and till date, only two cases are reported in literature ${ }^{2,3}$. Here, we present a case of an adult male who developed IPA 2 years posttransplant while not on immunosuppressive therapy for 406 days.

\section{Case Report}

A 43 years old male presented with gradually progressive dyspnoea and cough for 2 years which worsened since 10 days prior to admission. The patient was diagnosed as a case of acute promyelocytic leukaemia (APML) in March 2010 and treated with arsenic trioxide (ATO). After a year, the relapse was noted and again treated with ATO, mitoxantrone and all-trans retinoic acid (ATRA) for 45 days and maintenance therapy was given for 28 days and 10 days in a month for the next 12 months. After one year of completion of therapy, he was hospitalized with a complaint of severe headache and diagnosed as relapsed APML with leukemic meningitis. He was then treated with cytarabine, hydrocortisone, and methotrexate (TIT regimen) twice weekly till three serial 
lumbar punctures were cleared off abnormal cells. After placement of central venous catheter, the patient was put on 7,3 induction (cytarabine, daunomycin) with ATRA (22.04.13). During the therapy, the patient developed highgrade fever and blood tinged expectoration with decreased leucocyte counts (TLC $2400 / \mathrm{mm}^{3}$ ) which didn't respond to parenteral antibiotics. HRCT thorax revealed focal ground glass infiltrates and he was started on antifungal treatment (voriconazole parenteral $200 \mathrm{mg}$ twice a day for one week than oral for next eight weeks). He was conditioned with busulfan and cyclophosphamide and underwent sibling matched allogenic stem cell transplant (06.06.13). The patient received cyclosporine, prednisolone as immunosuppressant and prophylaxis for Pneumocystis jirovecii and Cytomegalovirus (CMV) for next 10 months post-transplant. The patient also had history of bronchial asthma 15 years before for which he received inhaled bronchodilators and corticosteroids for 5 years. After change in habitation, he became asymptomatic and didn't receive any treatment for it.

At the time of admission, the patient was tachypnoeic (respiratory rate of 32 breaths per minute) and had tachycardia (heart rate 124 beats per minute) with resting oxyhemoglobin saturation of $84 \%$, with use of accessory muscles of respiration. Auscultation revealed diffuse coarse crepitations and rhonchi over bilateral lung fields. Arterial blood gas analysis showed hypercapnic respiratory failure with hypoxemia ( $\left.\mathrm{pH} 7.30 ; \mathrm{pO}_{2} 56.2 ; \mathrm{pCO}_{2} 60 ; \mathrm{HCO}_{3} 26\right)$. Non-invasive ventilation (NIV) was given but the patient's blood gas measurements progressively deteriorated and then he was intubated and put on invasive mechanical ventilation.

Laboratory investigation showed total leucocyte count (TLC) and absolute neutrophil count (ANC) 26,500/ $\mathrm{mm}^{3}$ and $20,644 / \mathrm{mm}^{3}$, respectively. C-reactive protein (CRP) was elevated $(202.3 \mathrm{mg} / \mathrm{dl})$ with mild increased procalcitonin level $(0.28 \mathrm{ng} / \mathrm{dl})$ and normal liver and kidney function tests. The patient's condition and oxygenation deteriorated despite the use of broad-spectrum parenteral antibiotics. Chest radiograph showed bilateral patchy infiltrates. A high resolution computed tomography (HRCT) scan of the thorax that was done revealed multiple nodular lesions with perilesional ground glass opacity (GGO) distributed on both lung fields (Figure 1). Peribronchovascular thickening was also noted with focal areas of bronchiectasis at the left lower lobe.

Based on HRCT scan thorax, an empirical antifungal therapy (Parenteral Voriconazole $200 \mathrm{mg}$ twice a day) was started and endotracheal aspirate was sent to microbiology laboratory for microbial cultures as well as serum galactomannan assay was carried out. Aerobic culture and sensitivity demonstrated the growth of Acinetobacter spp. which was sensitive only to polymyxin B.

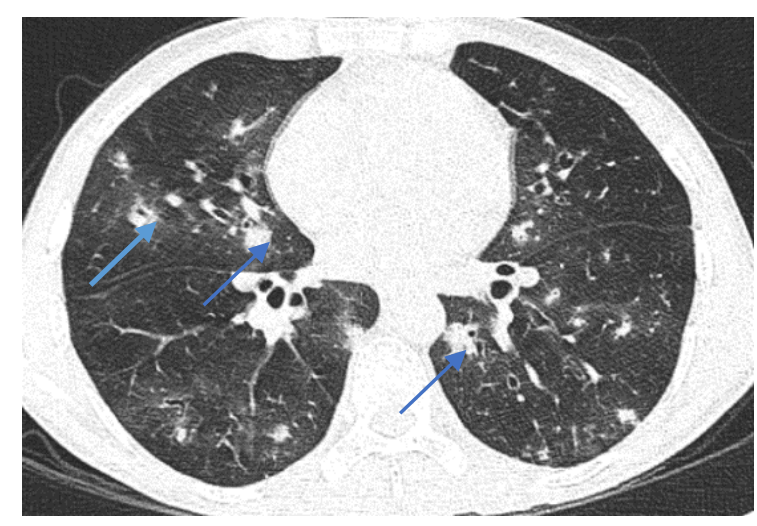

Figure 1: High resolution computed tomography (HRCT) thorax (19.02.16) revealed multifocal small nodular lesions with Perilesional ground glass opacity (Halo sign); blue arrow.

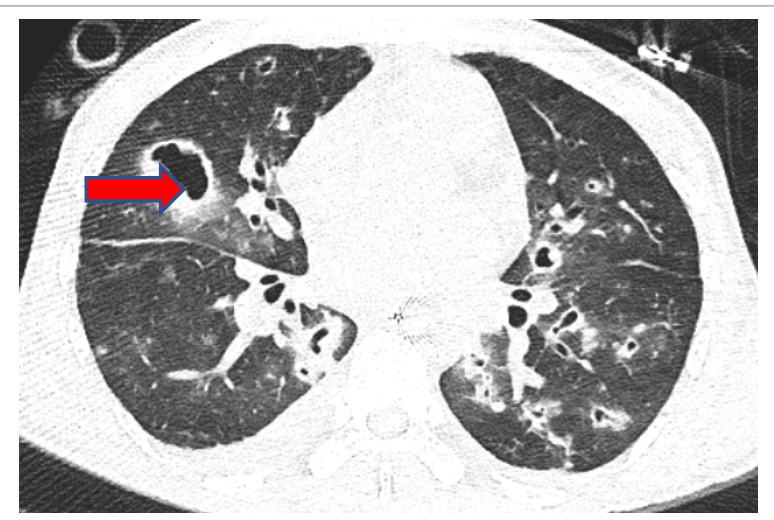

Figure 2: HRCT thorax (04.03.16) showed progression of IPA with cavitation (red arrow).

Serum galactomannan test was positive and fungal culture revealed the growth of Aspergillus fumigatus.

These results confirmed the diagnosis of IPA. Antifungal therapy was continued, and antibiotic regimen was also upgraded to meropenem, colistin and teicoplanin. The patient was evaluated for immunodeficiency and the immunoglobulin levels were within normal limits (IgG, IgM, IgA; 1080, 129, $247 \mathrm{mg} / \mathrm{dl}$ respectively). The patient's condition was largely improved with a decrease in leukocyte counts and CRP levels and reduced ventilatory requirement, but this was punctuated by frequent episodes of clinical worsening. Repeated CT thorax revealed the progression of IPA with the formation of multiple large cavitary lesions (Figure 2). Caspofungin was added after 2 weeks of voriconazole as there was radiological progression of the IPA. However, the patient died after 40 days of ICU stay.

\section{Discussion}

Aspergillus spp. produce a wide spectrum of pulmonary diseases from non-invasive saprophytic syndromes to angio-invasive disease based on immune status of an 


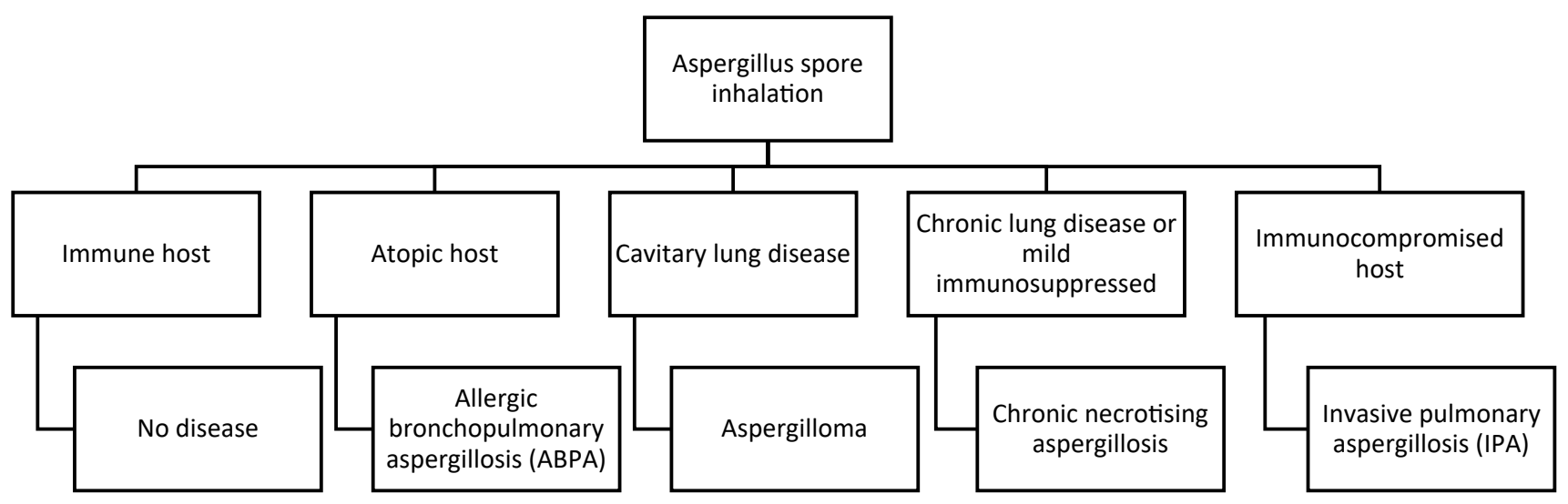

Figure 3: The clinical spectrum of pulmonary aspergillosis.

individual host (figure 3$)^{4}$. IPA is the most severe form of pulmonary disease with higher rates of morbidity and mortality. Incidence of IPA among the cases of HSCT is of $5-30 \%$ in allogenic and $1-5 \%$ in autologous transplant recipients $^{5,6}$. Rankin firstly described the case of IPA in a patient with agranulocytosis in $1953^{7}$. Nowadays, because of the widespread use of chemotherapy and immunosuppressant agents, the incidence has increased dramatically. The mortality rate among these cases are found to be more than $50 \%$ but it reaches to $90 \%$ in HSCT recipients $^{8,9}$.

The development of IPA in HSCT transplant recipients showed bimodal distribution. The first peak is usually seen within the first month after HSCT and is associated with neutropenia. The second peak is seen during treatment of graft versus host disease (GVHD) with immunosuppressive medications (78-112 days post-transplant) ${ }^{5}$. Development of IPA after this time frame in unusual. After one year, the development of IPA is rare and limited to only few cases reported in the literature. Baddley et al. reported one case of IPA 470 days after HSCT in his retrospective analysis of 94 patients who underwent allogenic bone marrow transplant ${ }^{2}$. Similarly, Rashid et al. reported a case of IPA after 10 years of HSCT and 5 years of stopping immunosuppression $^{3}$. In the present case, HSCT was done two years back and immunosuppressive therapy was also stopped 406 days back, so he was not supposed to be immunocompromised. Moreover, the immunoglobulin levels were also within normal limits which exclude the possibility of immunodeficiency.

IPA is diagnosed on the basis of criteria laid down by European Organization for Research on Treatment for Cancer/ Mycoses Study Group (EORTC/MSG) ${ }^{10}$. Our patient was categorized as a probable case as we have used tracheal aspirate for fungal culture and histopathology was not performed which is considered as a gold standard. However, the yield of Aspergillus fumigatus in the respiratory tract specimens of the patients underwent HSCT should not be considered as colonization as positive sputum has a positive predictive value of $80-90 \%$ for diagnosis of IPA ${ }^{11,12}$. Our diagnosis of IPA was further supported by positive result of serum galactomannan assay. Pfeiffer et al reported that galactomannan assay is more useful in a patient who underwent HSCT transplant than other cases with sensitivity and specificity of $71 \%$ and $89 \%$, respectively ${ }^{13}$.

Our patient presented with gradually progressive dyspnoea and cough since last two years which indicates possibility of development of chronic GVHD. According to National Institute of Health (NIH) criteria for the diagnosis of chronic GVHD, lung involvement is diagnosed on the basis of clinical symptom (dyspnoea) and spirometry only ${ }^{14}$. However, previous spirometry was not available, and we could not perform it because of the poor general condition of the patient. HRCT scan thorax revealed only bronchiectasis and there was no evidence of air trapping. Further, we did not perform autopsy of the patient so, the possibility of chronic GVHD couldn't be ruled out. Chronic GVHD can predispose the individual to the development of IPA.

Donor-derived immune reconstitution in HSCT recipients' takes months to years to maturate and makes host to be susceptible for various infections. Innate immunity, including epithelial barriers and phagocytes, usually recovers within weeks, and B-cell and $\mathrm{CD} 8^{+} \mathrm{T}$-cell counts recover in months. $\mathrm{CD} 4^{+} \mathrm{T}$-cell counts remain low for several years ${ }^{15}$, and their recovery is distinctly slow and depends on various factors such as age of the recipient, disease status, transplant type i.e. autologous versus allogeneic, HLA disparity ${ }^{16}$. Nowadays, donor lymphocyte infusion is being used as a measure to prevent opportunistic infections and GVHD. Adoptive transfer of anti-Aspergillus T-helper 1 cells might be a therapeutic option in patients with high risk of development of IPA ${ }^{17}$.

In conclusion, there is the possibility of the 
development of IPA in HSCT recipients beyond the period of immunosuppression. Further prospective studies are warranted to identify particular risk factors for the late development of IPA and to optimize the duration of prophylactic anti-fungal therapy. Adoptive transfer of functionally active Aspergillus specific T- cells may be an important intervention for prevention of IPA in such cases though its role is yet to be establish.

\section{References}

1. Kousha M, Tadi R, Soubani AO. Pulmonary aspergillosis: a clinical review. Eur Respir Rev. 2011 Sep 1; 20(121): 156-74.

2. Baddley JW, Stroud TP, Salzman D, et al. Invasive mold infections in allogeneic bone marrow transplant recipients. Clin Infect Dis Off Publ Infect Dis Soc Am. 2001 May 1; 32(9): 1319-24.

3. Rashid R, Denning DW. Invasive pulmonary aspergillosis 10 years post bone marrow transplantation: a case report. J Med Case Reports. 2009; 3: 26-26.

4. Hope WW, Walsh TJ, Denning DW. The invasive and saprophytic syndromes due to Aspergillus spp. Med Mycol. 2005 Jan 1; 43(Supplement_1): S207-38.

5. Fukuda T, Boeckh M, Carter RA, et al. Risks and outcomes of invasive fungal infections in recipients of allogeneic hematopoietic stem cell transplants after nonmyeloablative conditioning. Blood. 2003 Aug 1; 102(3): 827-33.

6. Marr KA, Carter RA, Boeckh $\mathrm{M}$, et al. Invasive aspergillosis in allogeneic stem cell transplant recipients: changes in epidemiology and risk factors. Blood. 2002 Dec 15; 100(13): 4358-66.

7. Rankin NE. Disseminated Aspergillosis and Moniliasis Associated with Agranulocytosis and Antibiotic Therapy. Br Med J. 1953 Apr 25; 1(4816): 918-9.

8. Ninin E, Milpied N, Moreau P, et al. Longitudinal study of bacterial, viral, and fungal infections in adult recipients of bone marrow transplants. Clin Infect Dis Off Publ Infect Dis Soc Am. 2001 Jul 1; 33(1): 41-7.

9. Wald A, Leisenring W, van Burik JA, et al. Epidemiology of Aspergillus infections in a large cohort of patients undergoing bone marrow transplantation. J Infect Dis. 1997 Jun; 175(6): 1459-66.

10. Ascioglu S, Rex JH, de Pauw B, et al. Defining opportunistic invasive fungal infections in immunocompromised patients with cancer and hematopoietic stem cell transplants: an international consensus. Clin Infect Dis Off Publ Infect Dis Soc Am. 2002 Jan 1; 34(1): 7-14.

11. Horvath JA, Dummer S. The use of respiratory-tract cultures in the diagnosis of invasive pulmonary aspergillosis. Am J Med. 1996 Feb; $100(2): 171-8$

12. Yu VL, Muder RR, Poorsattar A. Significance of isolation of Aspergillus from the respiratory tract in diagnosis of invasive pulmonary aspergillosis. Results from a three-year prospective study. Am J Med. 1986 Aug; 81(2): 249-54.

13. Pfeiffer CD, Fine JP, Safdar N. Diagnosis of invasive aspergillosis using a galactomannan assay: a meta-analysis. Clin Infect Dis Off Publ Infect Dis Soc Am. 2006 May 15; 42(10): 1417-27.

14. Filipovich $\mathrm{AH}$, Weisdorf $\mathrm{D}$, Pavletic $\mathrm{S}$, et al. National Institutes of Health consensus development project on criteria for clinical trials in chronic graft-versus-host disease: I. Diagnosis and staging working group report. Biol Blood Marrow Transplant J Am Soc Blood Marrow Transplant. 2005 Dec; 11(12): 945-56.

15. Seggewiss R, Einsele $H$. Immune reconstitution after allogeneic transplantation and expanding options for immunomodulation: an update. Blood. 2010 May 13; 115(19): 3861-8.

16. Auletta JJ, Lazarus HM. Immune restoration following hematopoietic stem cell transplantation: an evolving target. Bone Marrow Transplant. 2005 May; 35(9): 835-57.

17. Tramsen L, Koehl U, Tonn T, et al. Clinical-scale generation of human anti-Aspergillus T cells for adoptive immunotherapy. Bone Marrow Transplant. 2009 Jan; 43(1): 13-9. 